\title{
Granulocyte Infiltration and Expression of the Pro-Angiogenic Bv8 Protein in Experimental EL4 and Lewis Lung Carcinoma Tumors
}

\author{
Kan Jiang ${ }^{1}$, Hyeongil Kwak ${ }^{2}$, Giovanna Tosato ${ }^{3}$ \\ 1. Laboratory of Cellular Oncology, Center for Cancer Research, National Cancer Institute, Bethesda, MD, \\ Laboratory of Cellular Oncology, Center for Cancer Research, National Cancer Institute, Bethesda, MD 2. \\ Laboratory of Cellular Oncology, Center for Cancer Research, National Cancer Institute, National \\ Institutes of Health 3. Laboratory of Cellular Oncology, Center for Cancer Research, National Cancer \\ Institute, National Institutes of Health
}

$\square$ Corresponding author: Giovanna Tosato, tosatog@mail.nih.gov

Disclosures can be found in Additional Information at the end of the article

Received 12/26/2012

Review began 12/27/2012

Published 01/18/2013

๑) Copyright 2013

Jiang et al. This is an open access article distributed under the terms of the Creative Commons Attribution License CC-BY 3.0., which permits unrestricted use, distribution, and reproduction in any medium, provided the original author and source are credited.

\section{Abstract}

Although vascular endothelial growth factor (VEGF)-targeted therapies have shown efficacy in the treatment of certain advanced cancers, benefits to patients have been modest, which is attributed to tumor resistance to VEGF neutralization. Recent efforts to identify new targets to inhibit tumor angiogenesis have identified Bv8 (prokineticin 2), a myeloid cell-derived protein that promotes endothelial cell growth and tumor angiogenesis, but many mechanistic aspects of the pro-tumorigenic function of Bv8 are unclear. Here we demonstrate that CD11b+, Ly6C+, Ly6G+ granulocytes are the predominant cell source of Bv8 expression in bone marrow, spleen and in tumor tissues. Using granulocyte-deficient growth factor independence-1 (Gfi1)-null mutant mice and normal littermates, we found that EL4 lymphoma tumors grow significantly larger in the granulocyte and Bv8-deficient mutant mice in comparison to the normal mice that display abundant tumor-associated granulocytes and Bv8 expression. Conversely, Lewis lung carcinoma (LLC-1) tumors grew to a significantly greater size in the normal mice in comparison to the Gfi1-null mice, but normal granulocyte tumor infiltration was modest. Quantitative analysis of tissue vascularization showed that EL4 and LLC-1 tumors from normal and Gfi1mutant mice are similarly vascularized. These results confirm the critical contribution of the tumor microenvironment in determining the rate of tumor progression independently of tumor angiogenesis, and reveal some of the complexities of granulocyte and Bv8 functions in modulating tumor growth.

Categories: Internal Medicine, Oncology

Keywords: angiogenesis, g-csf, bv8, tumor, gfi1, granulocyte

\section{Introduction}

Angiogenesis, the process by which new vessels are formed through the sprouting of endothelial cells, is critical during development as it provides a means for supplying oxygen and nutrients to tissues with increased need [1]. In the adult, vessels are normally quiescent and sprouting is physiologically limited to specific sites, such as the ovaries and the placenta [1]. However, in a number of diseases sprouting angiogenesis resumes.In cancer, angiogenesis contributes to disease progression [2]. Since VEGF is a key endogenous stimulator of physiological and pathological angiogenesis, different approaches to targeting VEGF with 
antibodies and small molecules have gained approval as adjuvant therapies for certain cancers $[1,2]$. However, the therapeutic benefits have been modest as tumors either are resistant or rapidly develop resistance to anti-VEGF treatment. To clarify the underlying reasons and improve anti-angiogenic treatment of cancer, recent efforts have focused on a more fundamental understanding of the angiogenic process in physiology and disease [3-5], and the discovery of biochemical signals and pathways other than those directly dependent on VEGF $[1]$.

Myeloid cells, which to varying degrees infiltrate tumors, have been shown to promote tumor angiogenesis, and to mediate resistance to anti-VEGF treatment [1,6]. Efforts to clarify the underlying mechanisms resulted in the identification Bv8, also known as prokineticin 2, a 77 amino acids protein product of $\mathrm{Gr} 1+\mathrm{CD} 11 \mathrm{~b}+$ myeloid cells induced by granulocyte colony stimulatory factor (G-CSF) [6-9]. Its G-protein-coupled receptors, (endocrine gland-derived) EG-VEGFR-1 and -2, have been identified on a variety of cells, including endothelial cells, hematopoietic progenitors and mature blood cells [6,10-12]. Bv8 promoted the proliferation of endothelial cells in vitro and angiogenesis in vivo, and its effects were comparable to those induced by VEGF in selected cultures and tissues [11,12]. Neutralization of Bv8 or its inducer GCSF in tumor-bearing mice inhibited tumor growth and tumor angiogenesis [7-9]. However, the mechanisms by which G-CSF promotes Bv8 expression, the cellular source of Bv8 production within the broader Gr1+CD11b+ cell population, and the extent to which Bv8 contributes to tumor angiogenesis and tumor growth are incompletely defined. To address these questions, we took advantage of a genetic mouse model of granulocyte deficiency induced by the homozygous deletion of Growth factor independence-1 (Gfi-1), a transcriptional repressor that critically regulates granulocyte maturation from bone marrow myeloid precursors $[13,14]$.

\section{Materials And Methods}

\section{Mice}

All mice (Gfi1-/- mutants and WT Gf11+/+ littermates C57BL/6; 4-8 weeks of age) previously described [13, 32]; all mice were housed in the animal facilities at the National Institutes of Health; animal studies were approved by the NCI Institutional Animal Care and Use Committee and were conducted in accordance with institutional guidelines. G-CSF treatment was carried out as described previously [32].

\section{Tumor models}

The murine lymphoma EL4 cell line and the murine Lewis lung carcinoma cell line LLC-1 (from ATCC) $(10 \times 106$ in $0.1 \mathrm{~mL}$ PBS) were implanted subcutaneously in the left abdominal quadrant of 6- to10-week-old mice. Tumors were harvested, weighed, and processed for flow cytometry, cell sorting, histology and/or gene expression studies, as described [33].

\section{Cells}

Bone marrow cells were obtained by flushing femora and tibiae, as described [34]. Cells were obtained from single-cell suspended spleens and tumor tissues by standard techniques. Cells were sorted electronically (FACSVantage SE; BD Biosciences) from bone marrow, spleen and tumors after cell-surface staining with APC-CD11b (Biolegend), PE-Ly6C (BD Pharmingen) and FITC-Ly6G antibodies (BD Pharmingen).

\section{Tumor immunohistochemistry}

Tumors were fixed in 4\% PFA and cryopreserved. Tissue sections were stained with rat antimouse CD31 monoclonal antibody (BD Pharmingen, San Diego, CA). Nuclei were visualized with DAPI (1:2000; Invitrogen). Sections were imaged with an Axiovert 200 fluorescence 
microscope (Carl Zeiss, Thornwood, NY).

\section{Image quantification}

Images from fluorescence staining were analyzed using NIH ImageJ software (http://rsb.info.nih.gov/ij/download.html). Pixel values for CD31 and DAPI fluorescence were separately obtained from individual tumor sections to compose the entire section. A ratio of CD31 pixel values/DAPI pixel values was calculated from each section to derive a relative mean CD31 pixel count. Relative mean CD31 pixel counts from tumor sections of the same group were averaged.

\section{RNA isolation and measurement}

Total RNA extracted with TRIzol (Invitrogen) or Absolutely RNA nanoprep kit (Statagene) was reverse transcribed using high-capacity cDNA reverse transcription kit (Applied Biosystems). Bv8:

Sense: 5'GCA TGA CAG GAG TCA TCA TTT T 3'

Antisense: 5' AAA TGG CAG GAT ATC AGG AAA 3'

and GAPDH mRNAs were measured by real-time PCR using the double strand specific dye SYBR ${ }^{\circledR}$ Green system (Applied Biosystems), as described [35].

\section{Statistical analysis}

Group differences were evaluated by the two-tailed Student's t-test for sample sizes $\leqslant 5$. P values less than 0.05 were considered statistically significant.

\section{Results}

First, we examined the relative contribution of monocytes and granulocytes to the production of Bv8. Granulocytes are mature CD11b+ myeloid cells that also express the surface Ly6C and Ly6G antigens, whereas the monocytes are $\mathrm{CD} 11 \mathrm{~b}+$ myeloid cell that also express the surface Ly6C, but not the Ly6G, antigen [19]. 


\section{Cureus}

A

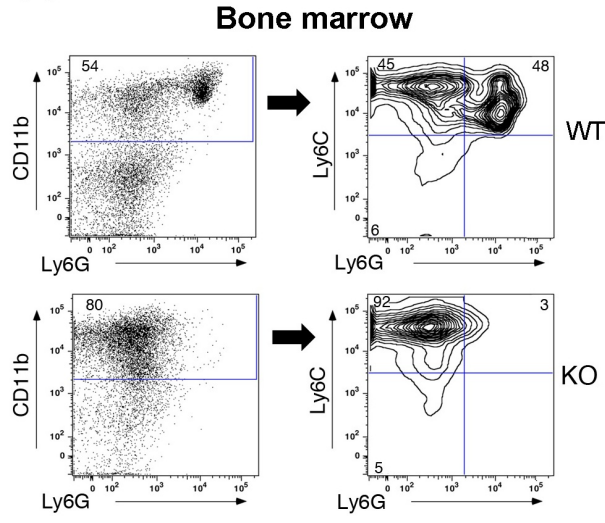

C

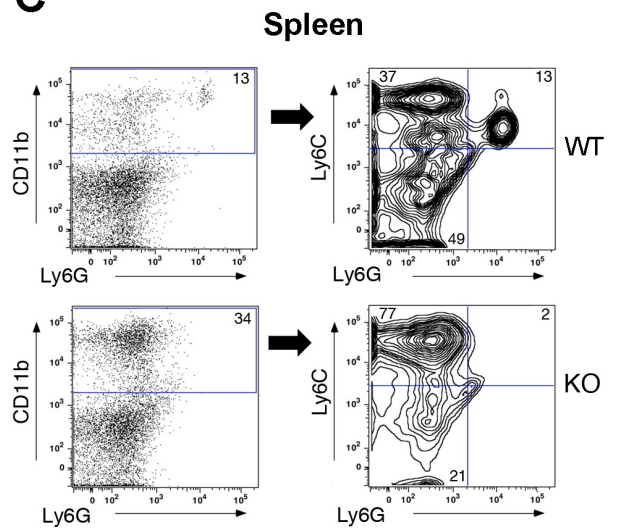

B
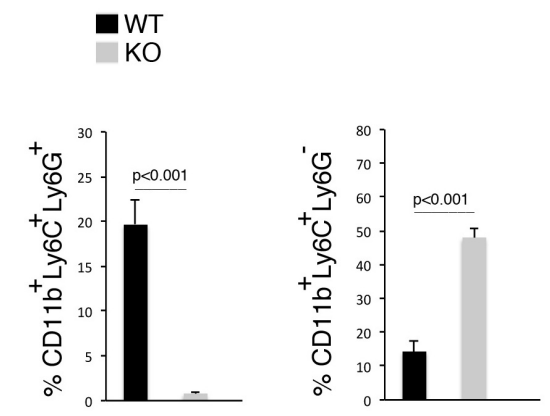

D

$$
\text { WWT }
$$

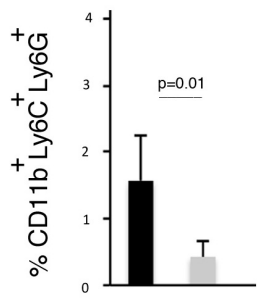

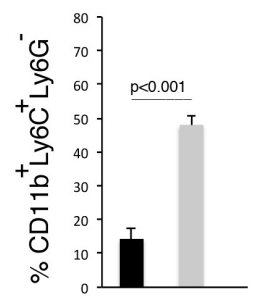

FIGURE 1: Relative distribution of granulocytes and monocytes in the bone marrow and spleen of Gfi1-null and normal littermate controls.

(A, C) Representative flow cytometry profiles. (B, D) Cumulative results from 3 mice each (means and SEM/group). The statistical significance of group differences ( $p$ ) is shown.

By flow cytometry, the bone marrow (Figures $1 A, 1 B$ ) and spleen (Figures $1 C, 1 D$ ) of Gfi1-null knock out mice (KO) clearly lack of CD11b+, Ly6C+, Ly6G+ granulocytes, whereas this population is normally represented in the wild-type (WT; Gfi1+/+) littermates (representative and cumulative flow cytometry profiles are shown). In addition, the bone marrow (Figures $1 \mathrm{~A}$, $1 B$ ) and spleen (Figures $1 C, 1 D$ ) of Gfi1-null mice display a relative increase of the monocyte CD11b+, Ly6C+, Ly6G- population. We sorted the monocytes (Ly6C+, Ly6G-) and the granulocytes (Ly6C+, Ly6G+) from the combined CD11b+ bone marrow cells of 5 WT mice (Figure 2A), and the monocytes (CD11b+, Ly6C+, Ly6G-) from the combined bone marrows of 5 Gfi1-null mice (Figure $2 B$ ); we could not sort the granulocytes from the granulocyte-deficient Gfi1-null bone marrows since the granulocyte gate was virtually empty (Figure $2 B$ ). 

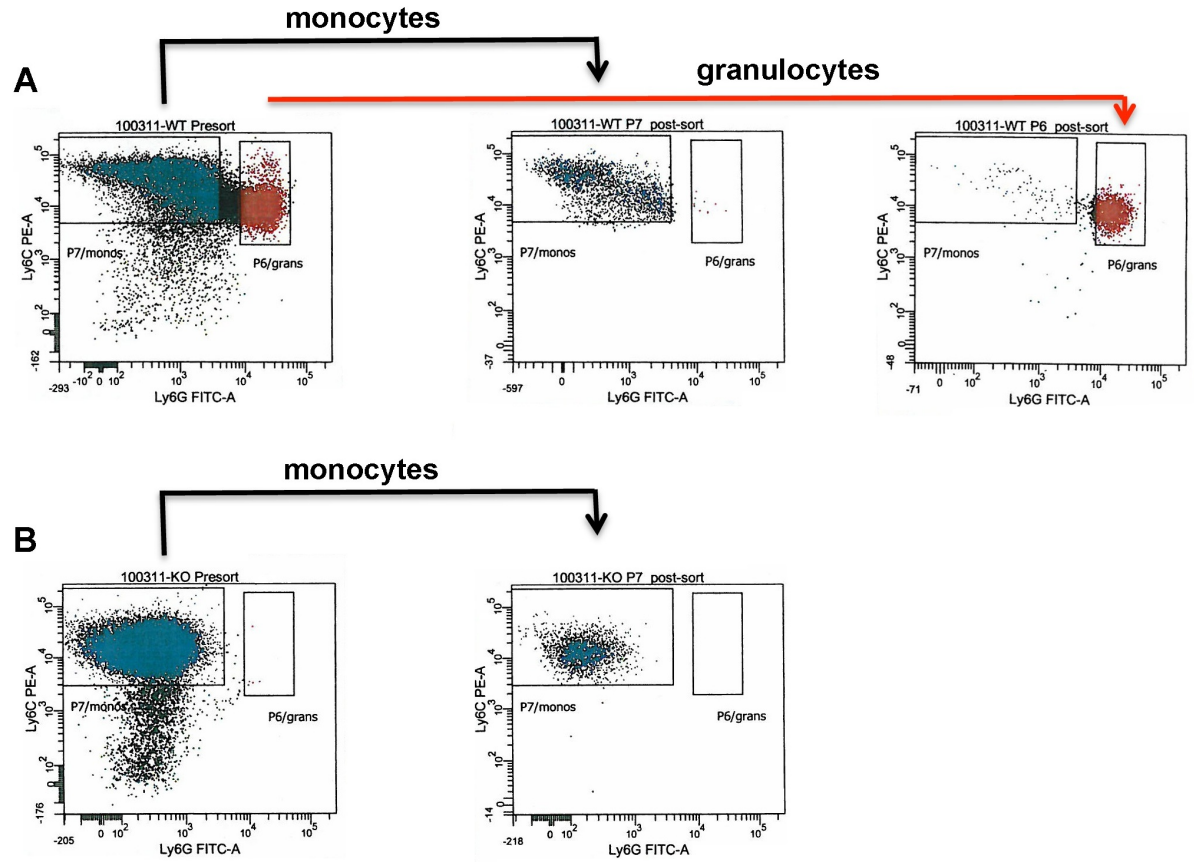

\section{FIGURE 2: Representative cell sorting profiles of monocytes and granulocytes from bone marrow.}

Bone marrows from 5 Gfi1-null and normal control littermates were labeled with antibodies for CD11b, Ly6C and Ly6G. After gating on CD11b+ cells (left panels), the Ly6C+Ly6G- monocytes and the Ly6C+Ly6G+ granulocytes were separated. (A) Normal WT control; (B) Gfi1-null.

We measured the levels of Bv8 mRNA expression in these cell populations by quantitative PCR (polymerase chain reaction). We found that constitutive levels of Bv8 mRNA were very low in all cell populations (Figure $3 A$ ). After G-CSF stimulation (10 ng/ml, 4 hr), Bv8 mRNA expression increased by over 700-fold in WT granulocytes and by about 8-fold in WT monocytes (Figure 3A). By contrast, Bv8 mRNA expression with G-CSF remained quite low in Gfi1-null monocytes (Figure $3 A$ ).

Based on these in vitro results, we measured Bv8 expression in WT and Gfi1-null mice in vivo. G-CSF (filgrastim; Amgen) was administered to groups of WT and Gfi1-null mice intraperitoneally (i.p.) daily for 5 days at the dose of $5 \mathrm{mg} / \mathrm{mouse}$. Consistent with the results in vitro, we found that G-CSF promotes Bv8 expression in WT bone marrow cells, whereas levels of Bv8 remained low in Gfi1-null bone marrows in spite of G-CSF administration (Figure 3B). These results indicate that constitutive levels of Bv8 expression are low in normal and Gfi1-null bone marrows; that G-CSF greatly stimulates Bv8 expression in the normal bone marrow cells but not in the Gfi1-null bone marrow cells; that bone marrow granulocytes, not monocytes, are the principal source of G-CSF-induced Bv8 expression. As shown in Figure 1, such granulocyte population is largely missing from Gfi1-null bone marrows, whereas previous studies have shown that monocytes are functionally normal in Gfi1-null mice [20]. The difference in Bv8 expression by WT monocytes and granulocytes is not attributable to G-CSF unresponsiveness by bone marrow monocytes because such cells express a functional G-CSF receptor (G-CSFR; Csf3r), which mediates critical biological effects of G-CSF in this population [21]. 


\section{Cureus}

A
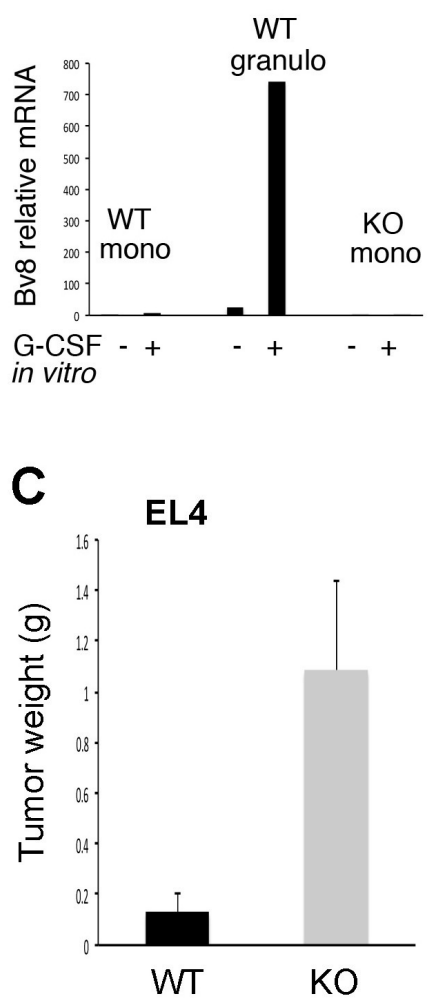

B

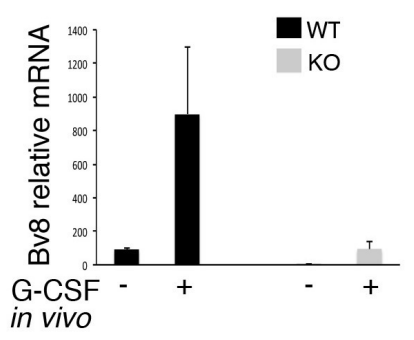

D LLC-1

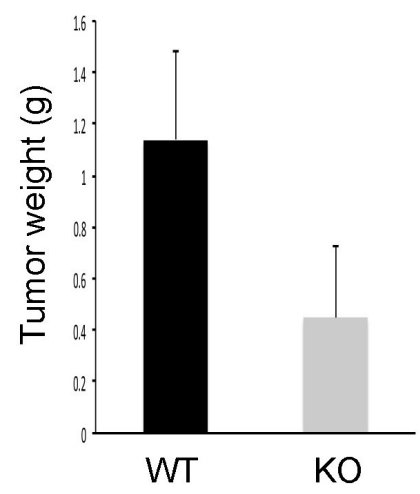

FIGURE 3: Analysis of Bv8 expression in bone marrow cell populations; tumor growth in normal WT and Gfi1-null KO mice recipients.

(A) Relative Bv8 mRNA expression in sorted populations of bone marrow WT monocytes (mono) and granulocytes (granulo) incubated in vitro (4hr) in either medium alone or medium supplemented with G-CSF (10 ng/ml). (B) The mice were first treated for 5 days with G-CSF (5 $\mathrm{mg} / \mathrm{mouse} /$ day) or left untreated; the bone marrow cells were then harvested and sorted into populations of monocytes and granulocytes. Relative Bv8 mRNA expression was measured in these cell populations. Groups of 5 mice (WT control littermates and Gfi1-null, KO) were injected subcutaneously with the mouse lymphoma EL4 (C) or the mouse lung carcinoma LLC-1 cell lines (D); tumors were removed on days 12 (EL4) or 15 (LLC-1) after injection and tumor weight was measured. The results reflect the group means $( \pm S D)$.

A previous study reported that administration of anti-Bv8 or anti-G-CSF neutralizing antibodies to mice injected subcutaneously (s.c.) with the murine lymphoma EL4 or Lewis lung carcinoma (LLC-1) cell lines resulted in a significant reduction of tumor angiogenesis and tumor growth, providing evidence that endogenous G-CSF and Bv8 contribute to EL4 and LLC1 tumor progression [8]. Based on the information shown in Figures 1-3, we predicted that experimental EL4 and LLC-1 tumors would grow less vigorously in Gfi1-null mice than in WT mice due to the granulocyte and Bv8 deficiency of Gfi1-null mice (Figures 1,2). To test this prediction, we injected subcutaneously (s.c.) groups of Gfi1-null mice and WT littermate controls with the syngeneic EL4 or LLC-1 cell lines. In a series of experiments, we found that s.c. injection of 10x106 EL4 or LLC-1 cells gave rise to progressively growing tumors in $100 \%$ of all (WT and GFi1-null) mice 5-7 days post injection. In contrast to our predictions, we found that EL4 tumors grew significantly $(\mathrm{p}<0.01)$ larger in the Gfi1-null mice compared to the 


\section{Cureus}

controls (Figure 3C). Instead, we found that LLC1 tumors grew significantly $(\mathrm{p}<0.01)$ larger in the WT controls than in the Gfi1-null recipient mice, which agreed with our predictions (Figure 3D).

Flow cytometric analysis of the inflammatory infiltrates in these tumors showed a significantly greater proportion of granulocytes (CD11b+, Ly6C+, Ly6G+) in EL4 tumors growing in the WT compared to Gfi1-null mice ( $\mathrm{P}<0.015$; Figure $4 \mathrm{~A}$ ). In contrast, granulocyte infiltration was similarly low in LLC-1 tumors even in WT and Gfi1-null mice ( $\mathrm{P}>0.05$; Figure $4 A)$. This analysis also showed a significantly greater monocyte (CD11b+, Ly6C+, Ly6G-) infiltration in LLC-1 tumors from Gfi1-null mice compared to the WT controls ( $\mathrm{P}<0.001$; Figure $4 B)$. Instead, monocyte infiltration was similar in EL4 tumors ( $>>0.05$; Figure $4 B)$. We detected no difference in the proportion of T cells infiltrating EL4 and LLC-1 tumors in WT and Gfi1-null mice (not shown).

A

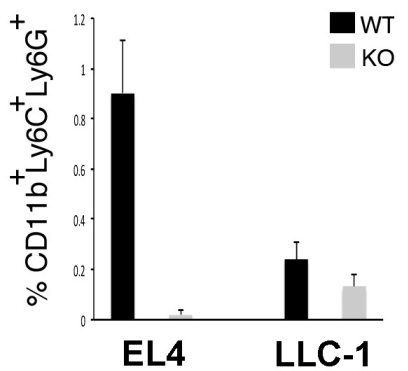

C

EL4-infiltrating

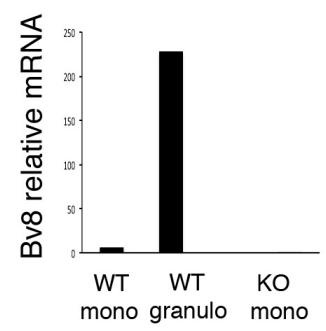

B

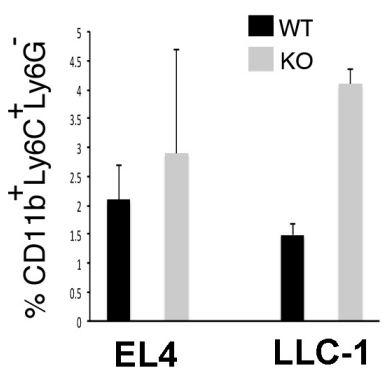

D

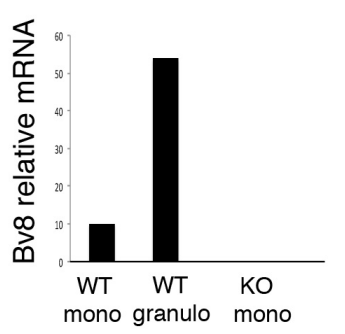

\section{FIGURE 4: Characterization of tumor-infiltrating cell populations and Bv8 expression by tumor-infiltrating granulocytes and monocytes.}

Granulocytes (A) and monocytes (B) were identified by flow cytometry from single cell suspensions of EL4 and LLC-1 tumors removed from groups (3-5) WT and KO mice. The results reflect the mean \% cell infiltration $( \pm S D)$. Monocytes and granulocyte populations were sorted from single cell suspensions of groups of EL4 (C) and LLC-1 (D) tumors from WT and KO mice; the results reflect the relative Bv8 mRNA levels in the WT and KO cell populations from the combined sorted cells. 


\section{Cureus}

mRNA (not shown), Bv8 expression was undetected in unfractionated EL4 and LLC-1 tumor tissues. Therefore, we sorted granulocyte and monocyte populations infiltrating these tumors. We found that WT granulocytes recovered from EL4 and LLC-1 tumors growing in WT mice expressed 5 to 25-fold higher levels of Bv8 mRNA compared to the WT monocytes recovered from these tumors, respectively (Figure $4 C, 4 D$ ). In addition, we found that Gfi1-null monocytes recovered from EL4 and LLC-1 tumors growing in Gfi1-null mice expressed virtually no Gfi1 mRNA (Figure 4C, 4D). As expected, we could not sort sufficient number of granulocytes from the tumors in the granulocyte-deficient Gfi1-null mice. Thus, the pattern of Bv8 expression in tumor-infiltrating populations is consistent with the pattern in bone marrow from non tumor-bearing mice (Figures 1,2) showing that WT granulocytes are a greater source of Bv8 compared to WT monocytes, and even more so than Gfi1-null monocytes.

These experiments indicate that EL4 tumor growth inversely correlated with granulocyte infiltration and tumor-associated Bv8 expression: tumors grew significantly more vigorously in Gfi1-null than in WT mice despite the significantly greater granulocyte infiltration and Bv8 expression in tumors arising in the Gfi1-null as opposed to WT mice. To examine whether increased LLC-1 tumor growth in WT mice compared to Gfi1-null mice was attributable to granulocyte infiltration and Bv8 expression, albeit modest by comparison to EL4 tumors, we examined tumor angiogenesis (Figure 5). Our analysis showed no significant differences $(\mathrm{P}>0.05)$ in the proportion of CD31+ endothelial cells detected in LLC-1 tumors growing in WT and Gfi1-null mice (Figure 5B), indicating that increased angiogenesis was unlikely responsible for increased LLC-1 growth in WT as opposed to Gfi1-null mice. A similar analysis in EL4 tumors also showed no significant difference $(\mathrm{P}>0.05)$ in tumor vascularization (Figure $5 A)$. Thus, granulocyte infiltration, expression of the pro-angiogenic Bv8 factor and tumor vascularization do not explain the differences in EL4 and LLC-1 tumor growth in normal mice and granulocyte-deficient Gfi1-null mice.

A
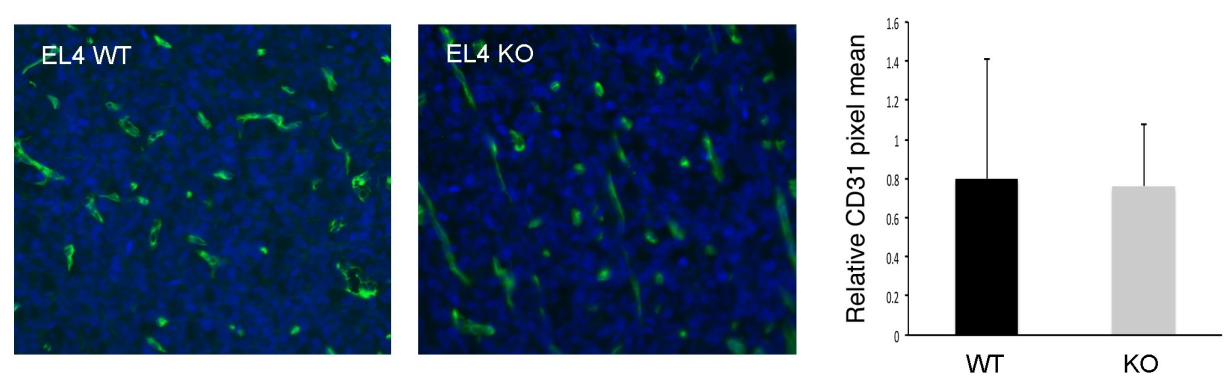

B
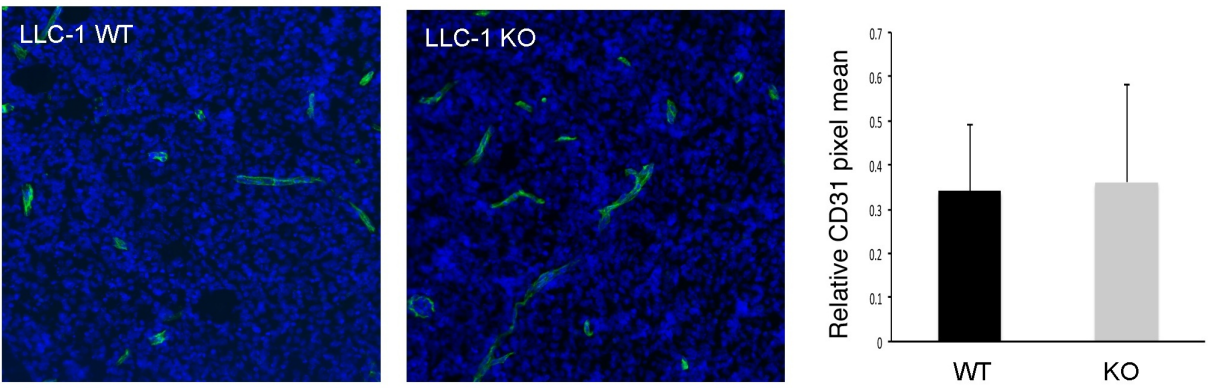

FIGURE 5: Analysis of tumor vasculature in EL4 and LLC-1 tumors arising in Gfi1-null and WT control mice.

Tumor sections from WT and KO mice were immuno-stained for the endothelial cell marker 
CD31 (green); the nuclei are stained with DAPI (blue). Representative images (left), and quantitative analysis of tumor vasculature (right) in EL4 (A) and LLC-1 tumor tissues (B) from WT and KO mice.

\section{Discussion}

Neovascularization is a hallmark of tumor cells [22], most of which secrete VEGF, a principal stimulator of endothelial cell sprouting angiogenesis [23, 24]. Not surprisingly, VEGF has emerged as the prime target for anti-angiogenic cancer therapy [2,25], based on the premise that reducing tumor vascularization may reduce tumor growth [26]. Several drugs that target VEGF have been developed, and some have proved to be efficacious as anti-cancer agents and have gained FDA approval [1, 2]: the VEGF neutralizing antibody bevacizumab (Avastin) for adjuvant therapy of several advanced cancers and as a single agent for the treatment of recurrent glioblastoma; tyrosine kinase inhibitors that target VEGFR2 and other receptors for adjuvant therapy of metastatic renal cell carcinoma (Sunitinib, Sorafenib, and Pazopanib) advanced liver (Sorafenib), medullary thyroid (Vandetanib) and neuroendocrine pancreatic (Sunitinib) cancers; and VEGF-trap (Aflibercept, Zaltrap) that binds VEGF-A and other VEGF family members for adjuvant therapy of advanced colorectal cancer.

Patient benefits from drugs that target VEGF is measured in weeks or months of increased cancer survival or progression-free survival. Mechanistically, VEGF-targeted therapeutics reduce tumor neovascularization by blocking the chaotic endothelial cell sprouting typical of many tumors; by inducing the regression of newly formed tumor vessels; and possibly by producing a functional "normalization" of the tumor vascular supply resulting in better delivery of chemotherapy $[1,2,27]$. It will be important to dissect the relative contribution of these different mechanisms to anti-angiogenic treatment of cancer, but much effort is currently placed in identifying new and improved targets. One such promising target is Bv8, identified as a product of tumor-infiltrating myeloid cells, which was shown to inhibit tumor angiogenesis even in the context of resistance to VEGF neutralization [6-9].

The studies presented here show that Bv8 is produced predominantly by granulocytes that reside in the bone marrow and the spleen or have infiltrated the tumor tissue, whereas other myeloid cells at these sites are a poor source of Bv8. Our studies also show that EL4 and LLC-1 tumors grow to significantly different sizes in normal and granulocyte-deficient mice, but whereas EL4 tumors grow more rapidly in the granulocyte/Bv8-incompetent KO mice, LLC-1 tumors grow more rapidly in the granulocyte/Bv8-competent mice. While confirming the critical importance of the tumor microenvironment as a determinant of tumor progression, the current results appear to contrast with those of previous studies showing that neutralization of Bv8 alone or with VEGF reduced substantially EL4 and LLC-1 tumor growth in mice [8]. The experimental conditions were somewhat different in that we used immunocompetent mice syngeneic to the cell lines, whereas the previous study used T-cell immunodeficient nude mice [8], which raises the possibility of a confounding contribution of T-cell immunity introduced in our experiments. Since broad deficiencies in T-cell immunity are uncommon in cancer patients, the current results from T-cell immunocompetent mice may be instructive for future Bv8 drug development. It is interesting that CD8+ T-cells can promote granulocyte anti-tumor activity [19], and that granulocytes can either enhance or inhibit tumor growth [28]. Mechanistically, granulocytes can secrete pro-angiogenic factors, including Bv8 [29] and matrix-degrading enzymes [30], which promote tumor angiogenesis and tumor growth. They can also be cytotoxic for tumor cells and inhibit tumor cell growth [31, 32].

Gfi1 is a transcriptional repressor that targets a variety of genes [33]. Not unexpectedly, besides being granulocyte deficient, Gfi1-null mice have been reported to have other defects 
including defective monocyte maturation into dendritic cells [20], defective self-renewal of hematopoietic stem cells [33], defective T-cell precursor development and maturation [34] and reduced B-cell number in the bone marrow [14]. Despite these deficiencies, adult Gfi1-null mice display only a modest reduction in lymphoid populations in blood and peripheral lymphoid organs, and no defects in antigen-driven $\mathrm{T}$ and $\mathrm{B}$ cell responses [35]. Nonetheless, it is possible that the Gfi1-null mice may not solely reflect the absence of granulocytes when challenged with tumor cells.

\section{Conclusions}

The current results confirm the critical importance of the tumor microenvironment as a determinant of tumor progression, reinforce the potential antitumorigenic role of granulocytes and reveal previously unappreciated complexities of the proangiogenic function of granulocytes and Bv8.

\section{Additional Information \\ Disclosures}

Human subjects: All authors have confirmed that this study did not involve human participants or tissue. Animal subjects: The NCI-Bethesda Animal Care and Use Committee Issued protocol number \# MB-068. Conflicts of interest: In compliance with the ICMJE uniform disclosure form, all authors declare the following: Payment/services info: This work was supported by the Intramural Research Program at NIH, CCR, NCI. Financial relationships: All authors have declared that they have no financial relationships at present or within the previous three years with any organizations that might have an interest in the submitted work. Other relationships: All studies were performed in accordance WITH the approved protocol.

\section{References}

1. Potente M, Gerhardt H, Carmeliet P: Basic and therapeutic aspects of angiogenesis. Cell [Research Support, Non-U.S. Gov't Review]. 2011, 146:873-87. 10.1016/j.cell.2011.08.039

2. Crawford Y, Ferrara N: VEGF inhibition: insights from preclinical and clinical studies . Cell Tissue Res. [Review]. 2009 , 335:261-9. 10.1007/s00441-008-0675-8

3. Sawamiphak S, Seidel S, Essmann CL, et al.: Ephrin-B2 regulates VEGFR2 function in developmental and tumour angiogenesis. Nature. 2010, 465:487-91. 10.1038/nature08995

4. Wang Y, Nakayama M, Pitulescu ME, et al.: Ephrin-B2 controls VEGF-induced angiogenesis and lymphangiogenesis. Nature. 2010, 465:483-6. 10.1038/nature09002

5. Gaengel K, Niaudet C, Hagikura K, et al.: The sphingosine-1-phosphate receptor S1PR1 restricts sprouting angiogenesis by regulating the interplay between VE-cadherin and VEGFR2. Dev Cell [Research Support, Non-U.S. Gov't]. 2012, 23:587-99. 10.1016/j.devcel.2012.08.005

6. Shojaei F, Wu X, Malik AK, et al.: Tumor refractoriness to anti-VEGF treatment is mediated by CD11b+Gr1+ myeloid cells. Nat Biotechnol. 2007, 25:911-20. 10.1038/nbt1323

7. Shojaei F, Singh M, Thompson JD, Ferrara N: Role of Bv8 in neutrophil-dependent angiogenesis in a transgenic model of cancer progression. Proc Natl Acad Sci . 2008, 105:2640-5. 10.1073/pnas.0712185105

8. Shojaei F, Wu X, Qu X, et al.: G-CSF-initiated myeloid cell mobilization and angiogenesis mediate tumor refractoriness to anti-VEGF therapy in mouse models. Proc Natl Acad Sci. 2009, 106:6742-7. 10.1073/pnas.0902280106

9. Shojaei F, Wu X, Zhong C, et al.: Bv8 regulates myeloid-cell-dependent tumour angiogenesis. Nature. 2007 , 450:825-31. 10.1038/nature06348

10. Lin R, LeCouter J, Kowalski J, Ferrara N: Characterization of endocrine gland-derived vascular endothelial growth factor signaling in adrenal cortex capillary endothelial cells. J Biol Chem. 2002, 277:8724-9. 10.1074/jbc.M110594200

11. LeCouter J, Lin R, Tejada M, et al.: The endocrine-gland-derived VEGF homologue Bv8 
promotes angiogenesis in the testis: Localization of Bv8 receptors to endothelial cells. Proc Natl Acad Sci U S A. [Research Support, Non-U.S. Gov't]. 2003, 100:2685-90.

10.1073/pnas.0337667100

12. LeCouter J, Zlot C, Tejada M, Peale F, Ferrara N: Bv8 and endocrine gland-derived vascular endothelial growth factor stimulate hematopoiesis and hematopoietic cell mobilization. Proc Natl Acad Sci U S A. 2004, 101:16813-8. 10.1073/pnas.0407697101

13. Karsunky H, Zeng H, Schmidt T, et al.: Inflammatory reactions and severe neutropenia in mice lacking the transcriptional repressor Gfi1. Nat Genet. [Research Support, Non-U.S. Gov't]. 2002 , 30:295-300. 10.1038/ng831

14. Hock H, Hamblen MJ, Rooke HM, et al.: Intrinsic requirement for zinc finger transcription factor Gfi-1 in neutrophil differentiation. Immunity [Research Support, Non-U.S. Gov't Research Support, U.S. Gov't, P.H.S.]. 2003, 18:109-20. 10.1016/S1074-7613(02)00501-0

15. De La Luz Sierra M, Gasperini P, McCormick PJ, Zhu J, Tosato G. : Transcription factor Gfi-1 induced by G-CSF is a negative regulator of CXCR4 in myeloid cells. Blood [Research Support, N.I.H., Intramural]. 2007, 110:2276-85. 10.1182/blood-2007-03-081448

16. Segarra M, Williams CK, Sierra Mde L, et al.: Dll4 activation of Notch signaling reduces tumor vascularity and inhibits tumor growth. Blood. [Research Support, N.I.H., Extramural Research Support, N.I.H., Intramural Research Support, Non-U.S. Gov't]. 2008, 112:1904-11. 10.1182/blood-2007-11-126045

17. Salvucci O, Jiang K, Gasperini P, et al.: MicroRNA126 contributes to granulocyte colonystimulating factor-induced hematopoietic progenitor cell mobilization by reducing the expression of vascular cell adhesion molecule 1. Haematologica. [Research Support, N.I.H., Intramural]. 2012, 97:818-26. 10.3324/haematol.2011.056945

18. Segarra M, Ohnuki H, Maric D, et al.: Semaphorin 6A regulates angiogenesis by modulating VEGF signaling. Blood. 2012, 120:4104-15. 10.1182/blood-2012-02-410076

19. Fridlender ZG, Sun J, Kim S, et al.: Polarization of tumor-associated neutrophil phenotype by TGF-beta: "N1" versus "N2" TAN. Cancer Cell. [Research Support, N.I.H., Extramural]. 2009, 16:183-94. 10.1016/j.ccr.2009.06.017

20. Rathinam C, Geffers R, Yucel R, et al.: The transcriptional repressor Gfi1 controls STAT3dependent dendritic cell development and function. Immunity [Research Support, Non-U.S. Gov't]. 2005, 22:717-28. 10.1016/j.immuni.2005.04.007

21. Christopher MJ, Rao M, Liu F, Woloszynek JR, Link DC: Expression of the G-CSF receptor in monocytic cells is sufficient to mediate hematopoietic progenitor mobilization by G-CSF in mice. J Exp Med. [Research Support, N.I.H., Extramural]. 2011 , 208:251-60. 10.1084/jem.20101700

22. Hanahan D, Weinberg RA : Hallmarks of cancer: the next generation . Cell [Research Support, N.I.H., Extramural Review]. 2011, 144:646-74. 10.1016/j.cell.2011.02.013

23. Ferrara N, Kerbel RS: Angiogenesis as a therapeutic target. Nature [Review]. 2005, 438:967-74. 10.1038/nature04483

24. Gerhardt H, Golding M, Fruttiger M, et al.: VEGF guides angiogenic sprouting utilizing endothelial tip cell filopodia. J Cell Biol [Research Support, Non-U.S. Gov't]. 2003, 161:116377. 10.1083/jcb.200302047

25. Kim KJ, Li B, Winer J, et al.: Inhibition of vascular endothelial growth factor-induced angiogenesis suppresses tumour growth in vivo. Nature. 1993, 362:841-4. 10.1038/362841a0

26. Folkman J: Tumor angiogenesis: Therapeutic implications. N Engl J Med. [Review]. 1971, 285:1182-6. 10.1056/NEJM197111182852108

27. Goel S, Duda DG, Xu L, Munn LL, Boucher Y, Fukumura D, et al. : Normalization of the vasculature for treatment of cancer and other diseases. Physiol Rev [Research Support, N.I.H., Extramural Research Support, Non-U.S. Gov't Research Support, U.S. Gov't, Non-P.H.S. Review]. 2011, 91:1071-121. 10.1152/physrev.00038.2010

28. Fridlender ZG, Albelda SM: Tumor-associated neutrophils: friend or foe?. Carcinogenesis [Review]. 2012, 33:949-55. 10.1093/carcin/bgs123

29. Murdoch C, Muthana M, Coffelt SB, Lewis CE: The role of myeloid cells in the promotion of tumour angiogenesis. Nat Rev Cancer [Research Support, Non-U.S. Gov't Review]. 2008, 8:61831. 10.1038/nrc2444

30. Pekarek LA, Starr BA, Toledano AY, Schreiber H: Inhibition of tumor growth by elimination of granulocytes. J Exp Med. [Research Support, Non-U.S. Gov't Research Support, U.S. Gov't, P.H.S.]. 1995, 181:435-40. 10.1084/jem.181.1.435 


\section{Cureus}

31. Hicks AM, Riedlinger G, Willingham MC, et al.: Transferable anticancer innate immunity in spontaneous regression/complete resistance mice. Proc Natl Acad Sci U S A. [Research Support, N.I.H., Extramural Research Support, Non-U.S. Gov't]. 2006, 103:7753-8. 10.1073/pnas.0602382103

32. Di Carlo E, Forni G, Lollini P, Colombo MP, Modesti A, Musiani P: The intriguing role of polymorphonuclear neutrophils in antitumor reactions. Blood [Research Support, Non-U.S. Gov't Research Support, U.S. Gov't, Non-P.H.S. Review]. 2001, 97:339-45.

10.1182/blood.V97.2.339

33. Hock H, Hamblen MJ, Rooke HM, et al.: Gfi-1 restricts proliferation and preserves functional integrity of haematopoietic stem cells. Nature [Research Support, Non-U.S. Gov't Research Support, U.S. Gov't, P.H.S.]. 2004, 431:1002-7. 10.1038/nature02994

34. Yucel R, Karsunky H, Klein-Hitpass L, Moroy T: The transcriptional repressor Gfi1 affects development of early, uncommitted c-Kit+ T cell progenitors and CD4/CD8 lineage decision in the thymus. J Exp Med [Research Support, Non-U.S. Gov't]. 2003, 197:831-44.

10.1084/jem.20021417

35. Hock H, Orkin SH: Zinc-finger transcription factor Gfi-1: versatile regulator of lymphocytes, neutrophils and hematopoietic stem cells. Curr Opin Hematol [Research Support, N.I.H., Extramural Review]. 2006, 3:1-6. 10.1097/01.moh.0000190111.85284.8f 